\title{
Discussion 2.2
}

Ranke (FRG): Clearly, growth hormone (GH) improves height velocity in Turner syndrome, and possibly it will cause a gain of several centimetres. What remains now is to define the optimal therapy for the Turner population. Perhaps you could both give us your ideas on how to optimize the treatment in future, and tell us what you are doing to correlate the individual response with poorly-defined variables in order to improve growth.

Wit (The Netherlands): First, as regards injection frequency, I believe that most people agree that daily injection is best. Secondly, the optimal dose of GH is not settled in my view. It appears that $3 \mathrm{IU} / \mathrm{m} 2 /$ day is as effective as $4 \mathrm{IU} / \mathrm{m} 2 /$ day. Dr. Takano showed that there is only a small difference for a lot more hormone. More research is still needed on dose. The other variable is age, and 16 years is probably the upper limit for treatment. Age seems to be more important than bone age.

Takano (Japan): I agree. Also, as I have shown, GH treatment alone might not suffice, so we should combine it with anabolic steroids and then, if there is not a substantial increase in height, oestrogen therapy may be added.

Wit: Yes, if oxandrolone is added from the age of about 8 or 9 years, the children grow taller earlier, so that the duration of GH therapy is shorter for the same final height. We are now involved in a study to investigate that particular aspect, that is, how much oxandrolone needs to be added for the optimal result.

Bourguignon (Belgium): Might we find an answer to your question by looking at individual variations, which might relate to factors independent of therapy, such as karyotype or height deficit.

Wit: We have studied this in great detail and found a correlation between the GH profile, or rather the frequency of the peaks in the profile, and height velocity increment.

Tanner (UK): Is there more growth in the younger patients? Is it important to start therapy when young?

Wit: Yes, the change in height SD score carries a negative correlation with age, but the change in height velocity does not.

Tanner: This happens because people use the change in height SD score as if it were a growth rate, but it is not. The relationship between the change in height SD score and height velocity is nonlinear; it is a second order, and a very complicated, curve.

Aarskog (Norway): If girls over 16 years only grow about $2 \mathrm{~cm}$ in the first year, is this sufficient to initiate a very expensive treatment?

Wit: No, over 16 years I would not recommend starting treatment. Under 16 years, the gain in height may be $3 \mathrm{~cm}$. Is this enough to justify the expense? The government departments responsible for reimbursement probably would not think so, but the patient or her mother probably would. It is a matter of perspective.

Knudtzon (Norway): Dr. Wit, the peak height velocity during puberty occurred earlier in your patients than in ours. When we introduced oestradiol in our patients at a very low dose, they experienced a growth spurt. You said that some of your patients had already had oestradiol. Can 
you comment on this? Have you seen any data on untreated girls, and what is the response to GH like in girls who have not received oestrogen? Wit: In

another study, we have looked at the effect of oestrogens on growth. If low-dose oestrogen is started at a relatively early age, between 12 and 15 years, there is an increment in height velocity, just as in puberty. If oestrogen is started at a later age, this increment is no longer apparent, so it seems to be age-dependent. We have looked at girls given GH to see if height velocity increments were increased or decreased by the addition of oestrogen, and we cannot see any difference as yet.

Attanasio (FRG): Dr. Takano, you said that during therapy there was no increase in glucose intolerance. How was this established?

Takano: Glucose tolerance was normal in all patients before treatment. We excluded any girls with abnormal glucose tolerance before treatment.

Attanasio: What proportion was excluded for this reason?

Takano: About 10-15\%.

Price (UK): I wonder what your views are on compliance, and whether it is more difficult to estimate compliance in this group than in, for example, GH-deficient children. Have you tried analysing your data according to who gives the injection to see whether the response is better when the parents give the injections, rather than the patients themselves.

Takano: In Japan, the injections are, in most cases, done by the parents and compliance is very good. They are given a record book to tick each day, and they are very proud of their records. Chatelain (France): My question concerns the use of androgens in a clinical trial on female patients. I realize that everyone wants these girls to gain height and to improve their self-concept, but I am surprised that knowing what we know of the effects of androgens on many tissues, including the brain, that few people question the use of oxandrolone in a female. Personally, I am quite opposed to this, and I would like to hear the opinions of Dr. Takano and Dr. Wit.

Wit: We have some experience with oxandrolone as sole therapy. Many investigators used this in the 1970s in Turner syndrome and saw a height velocity increment, though no increase in final height. On the other hand, we did not see serious side-effects, even at the relatively high dose of $0.1 \mathrm{mg} / \mathrm{kg}$. I have since heard from others using low-dose oxandrolone, and the number of sideeffects seems to be very low. Now we are using low doses of 0.06 and $0.03 \mathrm{mg} / \mathrm{kg}$, and placebo in a three-arm study. We are looking at the lowest dose that still has an effect on the GH response, and we are monitoring voice fundamental frequency.

Takano: Personally, I do not treat patients with Turner syndrome with androgens or anabolic steroids. In Japan, we do not usually treat them with anabolic steroids. Some doctors have tried it, but patients have complained about side-effects, so we are very cautious. I agree with Dr. Chatelain's opinion. Further study will be required to clarify these problems.

Ranke: Although we are all aware that neither androgens nor oes-trogens are strangers to either sex, I share your concern, Dr. Chate-lain. We have been using this anabolic hormone for many years without knowing much about it.

Aarskog: In the 1960s and 1970s, we treated a group of patients with Turner syndrome with oxandrolone for long periods until they were 18 years of age. They also started oestrogen at 14 years of age. They gained only about 3 or $4 \mathrm{~cm}$ in height after 4 or 5 years of treatment. No sideeffects were observed apart from slight clitoral enlargement.

Bourguignon: I would like to question the timing of the onset of oestrogen therapy and final height. In the interest of optimizing the effects of $\mathrm{GH}$, we tended to delay starting oestrogen for as long as possible, but perhaps we should be reconsidering earlier attitudes. What are your 
attitudes and your recommendations on the use of oestrogen, bearing in mind that it may influence the total duration of GH therapy and the final height attained?

Wit: In The Netherlands, we have now decided that all girls with Turner syndrome should start oestrogen therapy at the age of 12 years. A retrospective study has shown that there is no difference in final height, whether oestrogen is started at that age or later.

Takano: In Japan, we do not have a standard age for starting oestrogen therapy, but if a patient has a growth velocity of less than $2 \mathrm{~cm} /$ year and is aged above 15 years, she will be given oestrogen.

Chipman (USA): There is one report in the literature covering two groups aged 13 and 19 years when oestrogen was started, and showing no difference in final height between the two groups. Dr. Takano, am I right in thinking that there is a sub- population in the Japanese cross-sectional study who had spontaneous puberty but were actually shorter?

Takano: The cross-sectional growth curves shown today were derived from 416 prepubertal patients with Turner syndrome. The patients who had undergone puberty were not included. Bourguignon: Another question is, what kind of oestrogen should be used? There are two points of concern: possible side-effects in terms of blood lipids and coagulation, and secondly, the recently demonstrated differences in action between oral oestrogens and transdermal oestrogens on the binding proteins of the hormones that are involved in growth. Have you any experience of this?

Wit: We have always given ethinyloestradiol so far, but we are in the process of changing to encapsulated ß-oestradiol for our next study.

Ranke: The advantage of transdermal therapy is more than compensated for by the fact that girls do not like it and the prevalence of local reactions is extremely high. Of the oral oestrogens, microencap-sulated $\beta$-oestradiol and oestradiol valerate probably have the advantage that treatment can easily be monitored by assaying blood oestradiol levels.

De Muinck Keizer (The Netherlands): I would like to add that Dr. Kastrup from Denmark has experience of the transdermal oestrogens from FRG and was disappointed by the level of oestrogens that reached the blood.

Wit: Another question that is also very important is, how quickly should the oestrogen dose be increased? It has been suggested today that after 6 months it should be doubled and after another 6 months, tripled. Perhaps it is preferable to maintain a low dose, so that the patients still have some development of secondary characteristics. Higher doses may indeed limit final height. Knudtzon: I know of no study that has shown any beneficial effect of adding low-dose oestradiol treatment to GH treatment on final height. We gave a very low dose of oestradiol for a whole year, and during this year, height increase was accompanied by a significant bone age advancement. Anyhow, it is probably more physiological to start with a low dose and increase it gradually. 